\title{
Molecular Characterization, 3D Modeling of Grass Carp Interleukin-10 Receptor 1 (IL10R1)
}

\author{
He Wei, Shangnian Wang, Lei Qin, Xinyan Wang, Hong Zhou \\ School of Life Science and Technology, University of Electronic Science and Technology of China, \\ Chengdu, China \\ Email: zhouhongzh@uestc.edu.cn
}

Received June 2013

\begin{abstract}
Interleukin-10 (IL-10) is an important cytokine that plays a pivotal role in natural and adaptive immune systems. However, in lower vertebrates, especially in teleost the receptor of this cytokine is still largely unknown. This paper described the cloning and characterization of grass carp interleukin-10 receptor 1 (gcIL10R1) and the 3D structure of its extracellular domain was predicted. The gcIL10R1 cDNA included 180 bp 5' untranslated region (UTR), 870 bp 3' UTR and an open reading frame (ORF) of $1632 \mathrm{bp}$. The ORF was found to encode a 543 amino acid protein with a putative JAK1 binding site, one STAT3 binding site. The phylogenetic analysis clusters gcIL10R1 with other teleost IL10R1s but independently of the amphibian, avian and mammalian IL10R1s. The 3D structure of its extracellular domain was the first homology model of a fish IL10R1 that revealed a high similarity with its mammalian and avian counterparts.
\end{abstract}

Keywords: Grass Carp; Interleukin-10 Receptor 1; Structure Characterization; 3D Modeling

\section{Introduction}

Interleukin-10 (IL-10) was first described as an activity production by mouse Th2 cells that inhibited activation and cytokine production by Th1 cells [1]. Since its discovery, the biological effects of IL-10 were intensively investigated in mammals. Known as a cytokine inhibitory factor, the predominant function of IL-10 is regulating immune responses through direct or indirect effects on many cell types, including macrophage/monocytes, $\mathrm{T}$ cells, B cells, APCs, and NK cells [2-4]. IL-10 inhibits the release of pro-inflammatory cytokines like TNF- $\alpha$, IL-1 $\beta$, IL-6, and IL-8 from monocytes/macrophage $[5,6]$. Moreover, IL-10 inhibits IL-12 synthesis in dentritic cells [7]. IL-10 also deduces the secretion of IL-23 by macrophage and IFN- $\gamma$ production in Th1 cells [8,9]. Our previous study has identified grass carp IL-10 and investigated its function in grass carp peripheral blood leukocytes [10].

IL-10 signaling initiates by binding of the ligand to its specific cell surface receptor. In mammals, the IL-10 receptor complex is composed of two different chains, IL10R1 and IL10R2. IL10R1 is the ligand-binding subunit and IL10R2 is the accessory subunit which helps IL-10 signaling transduction. IL10R2 is also part of receptor complexes of other ligands [11]. IL10R1 was first be cloned in 1993 [12]. It belongs to the class II cytokine receptor family whose extracellular domain form fibronectin type III domain that has several conserved amino acid positions that are important for their secondary structure [13]. The fibronectin type III domain also exists in the extracellular region of mammalian IFN- $\gamma$ receptor alpha chain [14]. When IL-10 binds to its receptor complex, JAK-STAT signaling pathway is activated and signaling transducer of activation 3 (STAT3) is phosphorylated by Janus kinases, JAK1 and Tyk2 [15].

IL-10 genes have been isolated and characterized from many non-mammalian species, like chicken (Gallus gallus) [16], frog (Xenopus tropicalis) [17], fugu (Fugu rubripes) [18], common carp (Cyprinus carpio L.) [19], rainbow trout (Oncorhynchus mykiss) [20], zebrafish (Danio rerio) [21] and grass carp [10] by comparative genomic analysis or by PCR-mediated homology cloning. Increasing number of reports described fish IL-10, but little is known about the IL-10 receptors in teleost. To date, IL10R1 has only been identified in zebrafish and goldfish [22] by gene synteny analysis.

In this investigation, IL10R1 cDNA has been identified and characterized from grass carp. The 3D model of extracellular domain of gcIL10R1 protein was predicted, representing the first $3 \mathrm{D}$ analysis of the quaternary structure of a fish IL10R1, which provides the basis for further investigation of the IL-10 signaling in teleost. 


\section{Material and Methods}

\subsection{Animals}

The 1-year-old Chinese grass carp (Ctenopharyngodon idellus) with 1 - $1.5 \mathrm{~kg}$ body weigh were obtained from Chengdu Tongwei Aquatic Science and Technology Company. An acclimation period of two weeks was observed prior to the experiments commencing. Tissues for cloning of IL-10R2 were taken from freshly killed fish according to the Regulation of Animal Use in Sichuan province, China.

\subsection{RNA Extraction and cDNA Synthesis}

Total RNA was extracted from grass carp spleen with Tripure Reagent (Roche, Basel, Switzerlan) according to the manufacturer's instructions. After extraction of total RNA, five $\mu \mathrm{g}$ was reverse transcribed to cDNA using Superscript II reverse transcriptase (Invitrogen, Carlsbad, CA) with oligo $d(T)_{18}$ primer.

\subsection{Cloning of Grass Carp IL10R1}

Partial cDNA sequence of the grass carp IL10R1 gene was obtained by PCR using the primers IL10R1F and IL10R1R (Table 1), which was designed basing on the conserved regions of goldfish and zebrafish IL10R1s. PCR was performed with one cycle of incubation at $94^{\circ} \mathrm{C}$ for $3 \mathrm{~min}$, followed by 35 cycles at $94^{\circ} \mathrm{C}$ for $30 \mathrm{~s}, 58^{\circ} \mathrm{C}$ for $30 \mathrm{~s}$ and $72^{\circ} \mathrm{C}$ for $1 \mathrm{~min}$ with a final cycle at $72^{\circ} \mathrm{C}$ for $10 \mathrm{~min}$. To obtain the complete grass carp IL10R1 sequence, 5' and 3' RACE techniques were used to obtain the 5' and 3'-end sequences of grass carp IL10R1 cDNA. Referring to the nucleotide sequence of the partial sequenced grass carp IL10R1 cDNA, gene-specific primers were designed for 3' and 5'-RACE (Table 1), and PCR was performed with a GeneRacer Kit (Invitrogen) according to the protocol.

\subsection{Sequence Analysis and Phylogeny Analysis}

The full cDNA sequence and deduced amino acid sequence of IL-10 were analyzed using BLAST program from NCBI and the ExPASy Molecular Biology server

Table 1. Oligonucleotide primers used to amplify grass carp IL10R1.

\begin{tabular}{ccc}
\hline Name & Sequence (5’to 3') & object \\
\hline IL10R1F & ATATGGGAGGGAATGTAACTG & partial \\
IL10R1R & TCCTGGTTTTGCACCACATGC & \\
IL10R1-5N1 & TCACCATCTTATCATCATCATC & \multirow{2}{*}{ 5'-race } \\
IL10R1-5N2 & CTTTCTGTGAATTTTAACCTTC & \\
IL10R1-3N1 & TCAGCAGTGGAAGAAGAAGC & 3'-race \\
IL10R1-3N2 & GATGGATATCGTAGTCAGAG & \\
\hline
\end{tabular}

(http://us.expasy.org). The multiple alighments were made using DNAMAN software (Lynnon Biosoft, PointeClaire, Canada). The molecular mass and isoelectric point of the putative grass carp IL10R1 were predicted using Compute PI/Mw tool at the ExPASy

(http://www.expasy.c-h/). The deduced signal peptide was predicted using SignalP [23] and the transmembrane regions were predicted using the TMHMM Server v. 2.0 (http://www.cbs.dtu.dk/services/T-HMM/). The fibronectin type III domain and the immunoglobulin-like fold of the grass carp IL10R1 were predicted using the InterPro Scan online server

(http://www.ebi.ac.uk/Tools/pfa/ip-rscan/). The putative $N$-glycosylation sites of the grass carp IL10R1 were determined using the NetNGlyc Server v. 1.0

(http://www.cbs.dtu.dk/-services/netnglyc/). Phylogenetic trees were constructed using MEGA3.1 software [24]. The genetic distance between species was calculated using $\rho$-distance method. The cladogram was generated using the neighbor-joining ( $\mathrm{NJ}$ ) method. In the analysis, the gaps were deleted, and a 1000 bootstrap procedure was used to test the robustness of the node on the trees.

\subsection{D Modeling}

Two suitable structural templates for grass carp IL10R1 extracellular domain, chicken IFN- $\gamma$ receptor alpha chain extracellular domains and human IFN- $\gamma$ receptor alpha chain extracellular domain were identified by a BLAST search as implemented in the SWISS-MODEL Protein Modelling Server (http://www.isb-sib.ch/). The automatic sequenc-e alignment thus obtained was used for homology modeling with SWISS-MODEL. The resulting theoretical model of a protein monomer was displayed and analyzed with Swiss-PDB Viewer. Models pictures were obtained using the PyMOL program (http://www.pymol.sourcefor-ge.net/index.html).

\section{Results}

\subsection{Molecular Cloning and Sequence Analysis of Grass Carp IL10R1}

By using the primers which were designed on the basis of the conserved region of the known fish IL10R1 sequences and 5' and 3' RACE, we got the cDNA sequences of grass carp IL10R1 (Figure 1). The gcIL10R1 shared 73\% homology with amino acid sequence of goldfish IL10R1, and gcIL10R1 protein had conserved four cysteine residues as well as partially conserved hydrophobic residues, suggesting importance in the structural integrity and receptor-ligand interactions. The gcIL10R1 protein had a putative fibronectin type III domain, an immunoglobin-like fold and several predicted $\mathrm{N}$-glycosylation sited. Its sequence also bared putative 


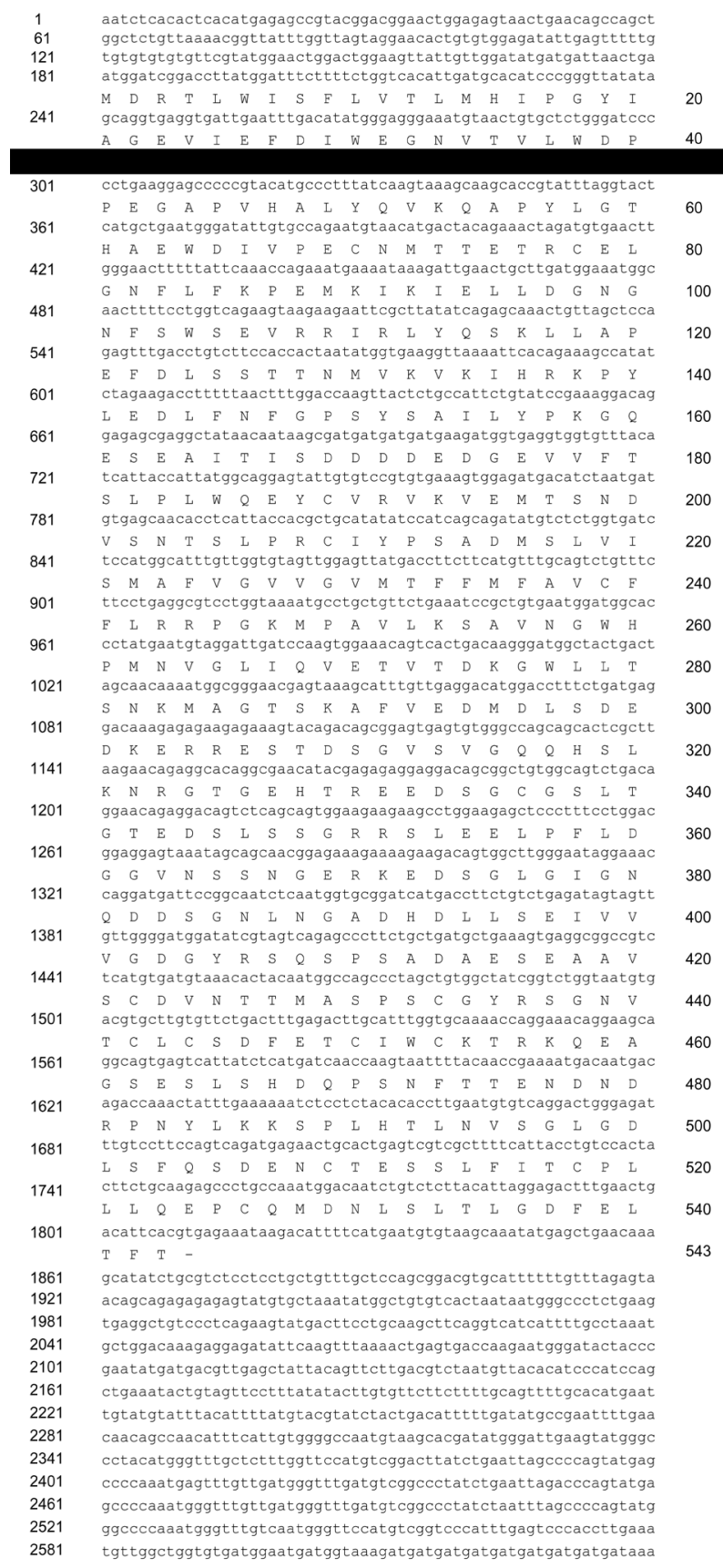

Figure 1. Nucleotide and deduced amino acid sequences of grass carp IL10R1.

JAK1 (SVLLFKK) and STAT3 (YXXQ) binding sites. The identified gcIL10R1 possessed one STAT3 binding site (Figure 2). Furthermore, like other fish, the C-terminal serine-rich areas were present in gcIL10R1 protein, which proposed to be essential for the immunosuppressive effects of the mammalian IL10R1 [25].

Phylogenetic analysis of gcIL10R1 proteins demonstrated their close relationship with the zebrafish and goldfish IL10R1s. The IL10R1 proteins of the above fish species branched independently to the IL10R1s of other vertebrates, while the amphibian and avian IL10R1s also branched independently from the IL10R1 of mammals (Figure 3).

\subsection{D Modeling}

The structure of gcIL10R1 extracellular domain which was for ligand binding was predicted by comparative modeling using the extracellular domains of chicken IFN- $\gamma$ receptor alpha chain (PDB id: 4EQ3) and human IFN- $\gamma$ receptor alpha chain (PDB id: 3S8W) as template. The homology model of grass carp IL10R1 has threehelix and several beta strands (Figure 4). The alignment used in the model shows a high similarity (75\%) between the target molecular and comparing extracellular domains of human and chicken IFN- $\gamma$ receptor alpha chain.

\section{Discussion}

In the present study, IL10R1 cDNA was cloned and characterized. For gcIL10R1, an open reading frame of 1632 bp was found to encode a 543 amino acid protein. The putative grass carp IL10R1 is a 60016 molecular mass polypeptide with a pI of 4.5. The gcIL10R1 shares high homology with amino acid sequence of other known teleost IL10R1. Analysis of gcIL10R1 sequence revealed the conserved putative JAK1 and STAT3 binding sites. JAK1 binding sites on the mammalian IL10R1 were identified in a region consisting of four core hydrophobic residues [26,27], comprising of the SVLLFKK motif on the human IL10R1. Our analysis of gcIL10R1 revealed that this region has been partially conserved evolutionarily, with gcIL10R1 proteins exhibiting the AVLKSAV motif in the corresponding region. The STAT3 binding motif (YXXQ), present on the mammalian IL10R1 is indispensable to IL-10 function [28,29]. This motif appears to have been evolutionarily conserved across vertebrate IL10R1 proteins including teleost. However, while the amphibian, avian and mammalian IL10R1 proteins possess two potential STAT3 binding sites, the grass carp and other teleost IL10R1 proteins appear to only have one putative STAT3 docking site. It will be interesting to learn the significance of having one rather than two STAT3 binding sites.

The predicted 3D structure for the gcIL10R1 extracellular domain revealed a high similarity with IFN- $\gamma$ receptor alpha chain. Since IL-10 has similar structure with IFN- $\gamma$ [14], our results indicated that gcIL10R1 is suitable for binding with grass carp IL-10.

Our studies cloned and analyzed the gcIL10R1 and its 3D structure was modeled. Those results suggested gcIL10R1 is structurally similar to its counterpart in mammals or avian, which pave the way for further investigation on IL-10 signaling in fish. 
grass carp.sec zebrafish.seq goldfish.seq frog.seq chicken.seq mouse.seq human.seq

grass carp.seq zebrafish.seq goldfish.seq frog.seq chicken.seq mouse.seg human. seq

grass carp.seq zebrafish.seq goldfish.seg frog.seq chicken.seg mouse.seq human.seq

grass carp.seq zebrafish.seq goldfish.seg

frog.seq chicken.seq mouse.seq human. seq

grass carp.seq zebrafish. seq goldfish.seq frog.seg chicken.seq mouse. seq human.seq

grass carp.seq zebrafish.seq goldfish.seq frog.seq chicken.seq mouse.seq human.seq

grass carp.seq zebrafish.seq goldfish.seq frog.seq chicken.seq mouse.seq human. seq

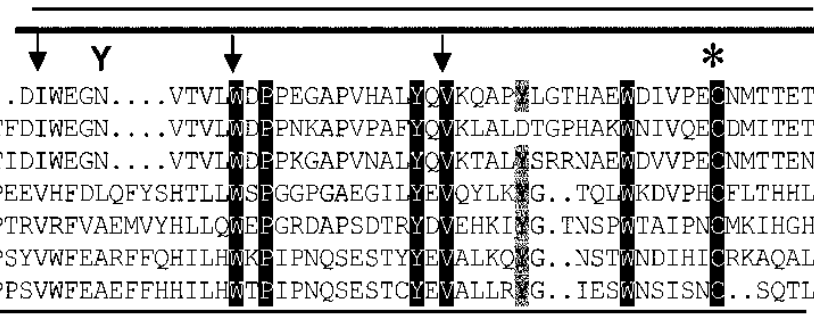

. MDRTLWISFVVTLMHIPGY IAGEVIEF . . . DIWEGN . . . VTVLW . . MDWTLWICEY⿱ $V$ VCCT . PGLIAGEQKKLNLTEDIWEGN . . . . VTVIN

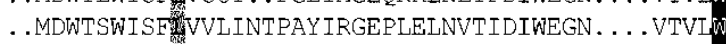
MAVLGASLLGT LCLSAFLHLGATEGGIPPAPEEVHEDLQEYSHTLLW .... MALCAA ALCVTLLLTQPAHGELRLKPTRVRFVAEMVYHLLQ ...MLSRLLPFWVTISSLSLEFIAYGTELPSPSYVWFEARFEQHILHV ...MLPCLVVI埥AALLSLRLGSDAHGTELPSPPSVWFEAEFFHHILHЙ

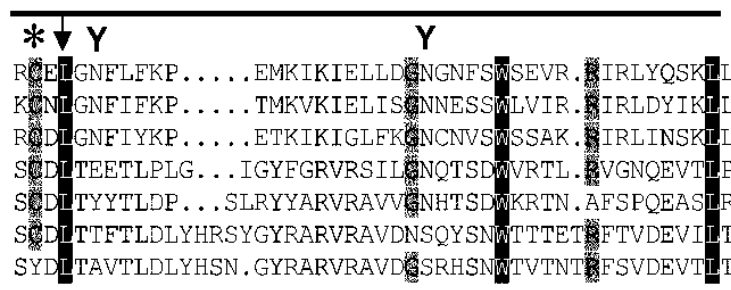

\section{$\downarrow \quad Y$}

\section{$\mathbf{Y} \downarrow$}

ISSTTNMVKVKIH , . RKPYLEDLENIGPSYSAI FSGPNTVKVKIH. RKPFLKELFPVGLSYSAI SESPNTVKVTIY . RKPELNDIFKXIGSMYSAM HVDGSSLLVDVSLPQTOAGNESLHYVDLFPFN AV'DNSIHVQLQ. LLLRAGNRTVKY DDIQKHA KAMDGIIYGTIHPPRPTITPAGDE EQVFKDL EIHNGEILGKIQLPRPKMAPANDT

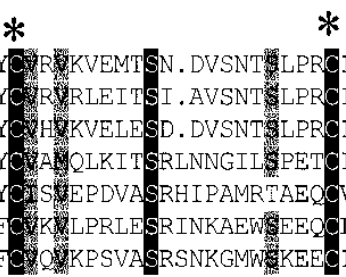

*

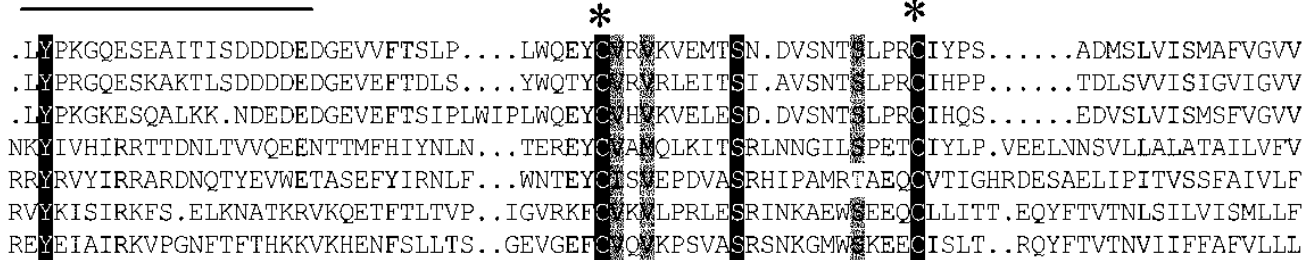

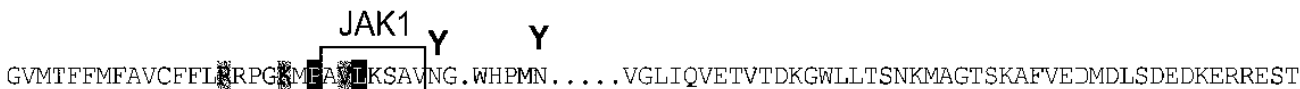
GVITLFMLFVCFFL RRPG MEA LKLAVNG. WLPMN . .....VGQTEVESVTQKGWLLNSNKIAEKTKAFDEIEELSEDEKERREST GVMSFFMFAICEFL RPG MFA'YKSAVNG.WNPMN . . . . V VGLIQVETVTDKGWLLTSNKKVEKSKEFDENKDLLEEDKERREST

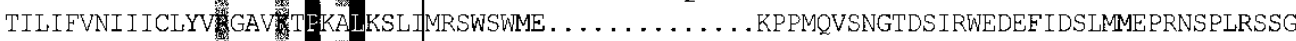
LLGILGALLVCTYL RPMMPFS IKSE IKQSSLWMEQ . . EISSSGSTDAVQQLFLCQKEPQHHGAPSTTGTAQLPTEKSSLLPAW CG. ILVCLVLQWYI HPG LTEWIVFKAPHDFEPAN. PLCPETPDAIHIVDLEVFPKVSLELRDSVLHGSTJSGFGSGKPSLTEE SGALAYCLALQLYV RRK

DSGVSVGQQHSLKNRGTGEHTREEDSGCG . .SLTGTEDSLSSGRRSLEELPFLDGGVNSSN.GERKEDAGLGIGNQDDSGNLNGAD DSGVSIGQQDSIKNR . ... PQREEDSGCG. . SLTGTEDSLSSGRRSLEELPFLDGGGNSSSVEGTRED GLGIQTQDISDK IKPMH DSGVSVGQQNSIKNEGLDGLTEQEDSGCG. . SLTGTEDSLSSGRRSLEELPFLDGEVNSSNESERKDD复GLGIGDQDASDNLTGTD DSGFGSQILTIKNSQ ............ LQSASLVLCIDDSGVELPESNSDCKKTFQNEDPTFKMHVPEIQAEDSGISLSTG PKEQAVGSRDSSCTSTDSGICLHSSSSSS... SSSSSSKLSHSENGGYKQQLPVGD. DSGVGLESPAG LIMEATHGAKPDFCPTTG SQFLLPGSHPQIQGTLGKEESPGLQATCG . . . DNTDSGICLQEPGLHSSMGPAWKQQLGYTHQDQDDWDVNLVQNSPGQPKYTQD PQFLLPDPHPQADRTLGNREPPVLGDSCSSGSSNSTDSGICLQEPSLSPSTGPTWEQQVGSNSRGQDD蔡GIJLVQNSEGRAGDTQG

\section{STAT3}

HDLLSEIVVVG. . . DG RRSDSPADAESEAAVSCDVNTTMASPSCGYRSGNVTCLCSDFETCIWCKTRKQEAGSES . LSHDQP.S DDLLSEIVVIG. . . DGYRSQSPSAEAETETT IQCDEDANMVSRTNGYRSGQVTCLCSDSETCMWCKTRKHLTDCDS . . FSHKQT . . VDLS. EIVVLG . . . DG RRSOSPSADAQSEAT IPCDIDANMASPSGGYRSGHVTCLCSDVETCMWCKTRKLLTDSDS . . LSHEQTSC SPCLKRSCSIQVCYGENTNGVRDNNCIEDVNDSRLGYLQdSEPKKNQNSPEMEEAEITQTKDYLQdVKQVLHIDNG . . I PRHNS. . QDSPDEVEFRG. YLQ SKGTVELRQGQADGGTLWACAGAPWGPAGTDIVLDMDCTELPVAKQYLKO SSPECPHSHTPALAAPGS. . ASALGHVCLIEPKAPEEKDQVMVTFQG YQKDTRWKAEAAGPAECLDEEIPLTDAFDPELGVHLQDDLAWPPPALAAGYLKESQGM GSALGHHSPPEPEVPGEEDPAAVAFQUYLRDTRCAEEKATKTGCLEEESPLTDGLGPKFGRCLVDEAGLHPPALAKGILKDPLEM

NETTENDNDRPNYLKKSPLHTLNVSGLGDLSFQSDENCT . ESSLFITCPLLLQEPCQMDNLS STLGDEELTE .... VNDNDRSSYLKKSPLETVNMFGLDDLSCHSDKTE . . ESSLFITCPLLLKEPYKLDTLP TLGGDVETT TETANNDNDRLNYLKKSPLDSVNVSGLEESSFQSEEDCG. . ESSLFITCPLMLQEPCELDILPEKLGDVKLTE .......NPQVQQEFFQGSWTNINEG.VHSSPPFTAAFS.... PFSRVLCDFRVNVPSLGDVQL ... . PMLTYGTSLASKASPELLKVP . .VDLGIEDTDLLG . TLPLISSLSSDEWLTVQTNSLSYLSRDSKDSR ASAPPGTPSRQWNQLTEEWSLLGVVSCEDLSIESWRFAHKLDPLDCGAAPGGLLDSLGSNLVT TISISSLQVE TLASSGAPTGQWNQPTEEWSLJALSSCSDLGISDWSFAHDLAPLGCVAAPGGLIGSFNSDLVT TISISSLQSS

Figure 2. Alignment of the amino acid sequence of grass carp IL10R1 with other homologues. The conserved cysteine residues are denoted by *. Conserved hydrophobic residues are denoted by arrows. The predicted glycosylation sites are denoted by overhead Y. The IL10R1 fibronectin type III domain and immunoglobin fold are indicated by an overhead line. Potential JAK1 and STAT binding sites are boxed. 


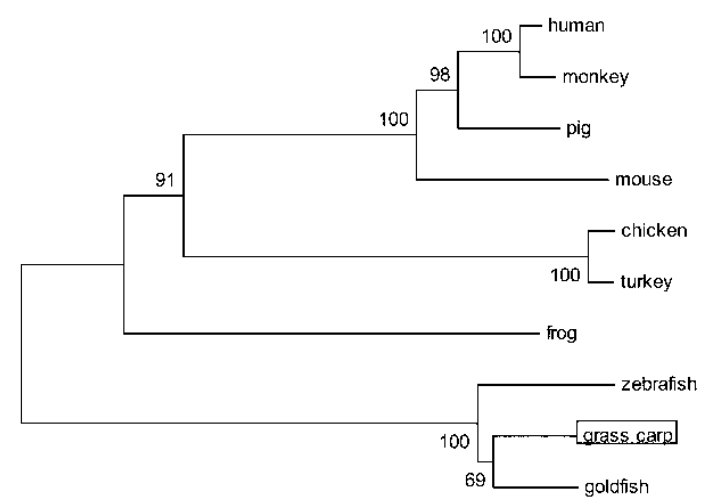

$\stackrel{\longmapsto}{0.1}$

Figure 3. Phylogenetic analysis of the IL10R1. An unrooted phylogenetic tree constructed by the neighbor joining method using MEGA software from the amino acid sequences of IL10R1s. Grass carp IL-10R1 was boxed. The numbers indicate the bootstrap confidence values obtained for each node after 1000 replications. The accession numbers of the sequences used in the alignment and in Figure 2 are as follows: Human IL10R1:NP_001549, Monkey IL10R1: XP_ 001092736, Pig IL10R1: XP_003129938, Mouse IL10R1: NP_032374, Frog IL10R1: AAI59332, Chicken IL10R1: NP_001034686, Turkey IL10R1:XP_003212786, Goldfish IL10R1: JN203498, Zebrafish IL10R1: NP_001071093, Grass carp: KF129394.

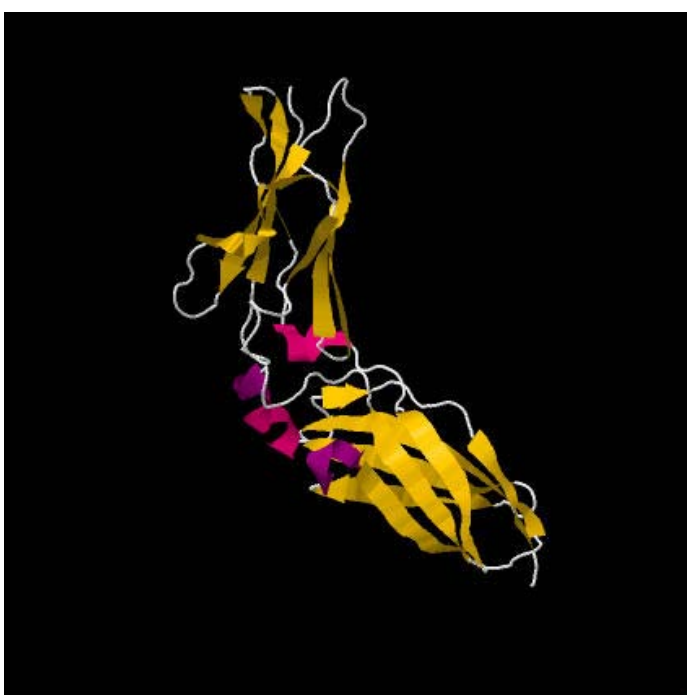

Figure 4. Homology modeling of grass carp IL10R1. Yellow arrows represent beta strands, red arrows represent alpha helices.

\section{Acknowledgements}

This work was supported by grant from the National Natural Science Foundation of China (31101877).

\section{REFERENCES}

[1] D. F. Fiorentino, M. W. Bond and T. R. Mosmann, "Two
Types of Mouse T Helper Cell. IV. Th2 Clones Secrete a Factor That Inhibits Cytokine Production by Th1 Clones,” The Journal of Experimental Medicine, Vol. 170, 1989, pp. 2081-2095. http://dx.doi.org/10.1084/jem.170.6.2081

[2] D. F. Fiorentino, A. Zlotnik, P. Vieira, T. R. Mosmann, M. Howard, K. W. Moore and A. O’Garra, “IL-10 Acts on the Antigen-Presenting Cell to Inhibit Cytokine Production by Th1 Cells,” The Journal of Immunology, Vol. 146, 1991, pp. 3444-3451.

[3] L. Ding and E. M. Shevach, "IL-10 Inhibits MitogenInduced $\mathrm{T}$ Cell Proliferation by Selectively Inhibiting Macrophage Costimulatory Function," The Journal of Immunology, Vol. 148, 1992, pp. 3133-3139.

[4] C. A. Akdis and K. Blaser, "Mechanisms of Interleukin10-Mediated Immune Suppression,” The Journal of Immunology, Vol. 103, 2001, pp. 131-136. http://dx.doi.org/10.1046/j.1365-2567.2001.01235.x

[5] R. de Waal Malefyt, J. Abrams, B. Bennett, C. G. Figdor and J. E. de Vries, "Interleukin 10(IL-10) Inhibits Cytokine Synthesis by Human Monocytes: An Autoregulatory Role of IL-10 Produced by Monocytes,” The Journal of Experimental Medicine, Vol. 174, 1991, 1991, pp. 12091220. http://dx.doi.org/10.1084/jem.174.5.1209

[6] D. F. Fiorentino, A. Zlotnik, T. R. Mosmann, M. Howard and A. O’Garra, "IL-10 Inhibits Cytokine Production by Activated Macrophages," The Journal of Immunology, Vol. 147, 1991, 1991, pp. 3815-3822.

[7] A. D’Andrea, M. Aste-Amezaga, N. M. Valiante, X. Ma, M. Kubin and G. Trinchieri, "Interleukin 10 (IL-10) Inhibits Human Lymphocyte Interferon Gamma-Production by Suppressing Natural Killer Cell Stimulatory Factor/ IL-12 Synthesis in Accessory Cells,” The Journal of Experimental Medicine, Vol. 178, 1993, pp. 1041-1048. http://dx.doi.org/10.1084/jem.178.3.1041

[8] A. Billiau and P. Matthys, "Interferon-Gamma: A Historical Perspective," Cytokine \& Growth Factor Reviews, Vol. 20, 2009, pp. 97-113.

http://dx.doi.org/10.1016/j.cytogfr.2009.02.004

[9] N. Schuetze, S. Schoeneberger, U. Mueller, M. A. Freudenberg, G. Alber and R. K. Straubinger, "IL-12 Family Members: Differential Kinetics of Their TLR4-Mediated Induction by Salmonella enteritidis and the Impact of IL-10 in Bone Marrow-Derived Macrophages,” International Immunology, Vol. 17, 2005, pp. 649-659. http://dx.doi.org/10.1093/intimm/dxh247

[10] H. Wei, M. Yang, T. Zhao, X. Wang and H. Zhou, "Functional Expression and Characterization of Grass Carp IL-10: An Essential Mediator of TGF-Beta1 Immune Regulation in Peripheral Blood Lymphocytes,” Molecular Immunology, Vol. 53, 2013, pp. 313-320. http://dx.doi.org/10.1016/j.molimm.2012.08.021

[11] G. Lutfalla, K. Gardiner and G. Uze, “A New Member of the Cytokine Receptor Gene Family Maps on Chromosome 21 at Less than $35 \mathrm{~kb}$ from IFNAR,” Genomics, Vol. 16, 1993, pp. 366-373. http://dx.doi.org/10.1006/geno.1993.1199

[12] A. S. Ho, Y. Liu, T. A. Khan, D. H. Hsu, J. F. Bazan and K. W. Moore, “A Receptor for Interleukin 10 Is Related 
to Interferon Receptors," Proceedings of the National Academy of Sciences of the United States of America, Vol. 90, 1993, pp. 11267-1171. http://dx.doi.org/10.1073/pnas.90.23.11267

[13] S. V. Kotenko, “The Family of IL-10-Related Cytokines and Their Receptors: Related, but to What Extent?” Cytokine \& Growth Factor Reviews, Vol. 13, 2002, pp. 223240. http://dx.doi.org/10.1016/S1359-6101(02)00012-6

[14] M. R. Walter, W. T. Windsor, T. L. Nagabhushan, D. J. Lundell, C. A. Lunn, P. J. Zauodny and S. K. Narula, "Crystal Structure of a Complex between InterferonGamma and Its Soluble High-Affinity Receptor,” Nature, Vol. 376, 1995, pp. 230-235. http://dx.doi.org/10.1038/376230a0

[15] R. M. Weber-Nordt, J. K. Riley, A. C. Greenlund, K. W. Moore, J. E. Darnell and R. D. Schreiber, "Stat3 Recruitment by Two Distinct Ligand-Induced, TyrosinePhosphorylated Docking Sites in the Interleukin-10 Receptor Intracellular Domain,” The Journal of Biological Chemistry, Vol. 271, 1996, pp. 27954-27961. http://dx.doi.org/10.1074/jbc.271.44.27954

[16] L. Rothwell, J. R. Young, R. Zoorob, C. A. Whittaker, P. Hesketh, A. Archer, A. L. Smith and P. Kaiser, "Cloning and Characterization of Chicken IL-10 and Its Role in the Immune Response to Eimeria maxima," The Journal of Immunology, Vol. 173, 2004, pp. 2675-2682.

[17] M. Chadzinska, E. Kolaczkowska, A. Scislowska-Czarnecka, N. Van Rooijen and B. Plytycz, "Effects of Macrophage Depletion on Peritoneal Inflammation in Swiss Mice, Edible Frogs and Goldfish,” Folia Biologica (Krakow), Vol. 52, 2004, pp. 225-231. http://dx.doi.org/10.3409/1734916044527557

[18] J. Zou, M. S. Clark and C. J. Secombes, “Characterisation, Expression and Promoter Analysis of an Interleukin 10 Homologue in the Puffer Fish, Fugu rubripes," Immunogenetics, Vol. 55, 2003, pp. 325-335. http://dx.doi.org/10.1007/s00251-003-0580-y

[19] R. Savan, D. Igawa and M. Sakai, “Cloning, Characterization and Expression Analysis of Interleukin-10 from the Common Carp, Cyprinus carpio L.," European Journal of Biochemistry, Vol. 270, 2003, pp. 4647-4654. http://dx.doi.org/10.1046/j.1432-1033.2003.03854.X

[20] Y. Inoue, S. Kamota, K. Ito, Y. Yoshiura, M. Ototake, T. Moritomo and T. Nakanishi, "Molecular Cloning and Expression Analysis of Rainbow Trout (Oncorhynchus mykiss) Interleukin-10 cDNAs," Fish and Shellfish Immunology, Vol. 18, 2005, pp. 335-344. http://dx.doi.org/10.1016/j.fsi.2004.08.004
[21] D. C. Zhang, Y. Q. Shao, Y. Q. Huang and S. G. Jiang, "Cloning, Characterization and Expression Analysis of Interleukin-10 from the Zebrafish (Danio rerion)," Journal of Biochemistry and Molecular Biology, Vol. 38, 2005, pp. 571-576.

http://dx.doi.org/10.5483/BMBRep.2005.38.5.571

[22] L. Grayfer and M. Belosevic, "Identification and Molecular Characterization of the Interleukin-10 Receptor 1 of the Zebrafish (Danio rerio) and the Goldfish (Carassius auratus L.),” Developmental \& Comparative Immunology, Vol. 36, 2012, pp. 408-417.

http://dx.doi.org/10.1016/j.dci.2011.08.006

[23] J. D. Bendtsen, H. Nielsen, G. von Heijne and S. Brunak, "Improved Prediction of Signal Peptides: SignalP 3.0," Journal of Molecular Biology, Vol. 340, pp. 783-795. http://dx.doi.org/10.1016/j.jmb.2004.05.028

[24] S. Kumar, K. Tamura and M. Nei, "MEGA3: Integrated Software for Molecular Evolutionary Genetics Analysis and Sequence Alignment," Briefings in Bioinformatics, Vol. 5, 2004, pp. 150-163. http://dx.doi.org/10.1016/j.jmb.2004.05.028

[25] J. Reboul, K. Gardiner, D. Monneron, G. Uze and G. Lutfalla, "Comparative Genomic Analysis of the interferon/Interleukin-10 Receptor Gene Cluster,” Genome Research, Vol. 9, 1999, pp. 242-250.

[26] A. Usacheva, R. Sandoval, P. Domanski, S. V. Kotenko, K. Nelms, M. A. Goldsmith and O. R. Colamonici, "Contribution of the Box 1 and Box 2 Motifs of Cytokine Receptors to Jak1 Association and Activation,” The Journal of Biological Chemistry, Vol. 277, 2002, pp. 4822048226. http://dx.doi.org/10.1074/jbc.M205757200

[27] A. Usacheva, S. Kotenko, M. M. Witte and O. R. Colamonici, "Two Distinct Domains within the N-Terminal Region of Janus Kinase 1 Interact with Cytokine Receptors," The Journal of Immunology, Vol. 169, 2002, pp. 1302-1308.

[28] A. M. O’Farrell, Y. Liu, K. W. Moore and A. L. Mui, "IL-10 Inhibits Macrophage Activation and Proliferation by Distinct Signaling Mechanisms: Evidence for Stat3Dependent and -Independent Pathways,” EMBO Journal, Vol. 17, 1998, pp. 1006-1018. http://dx.doi.org/10.1093/emboj/17.4.1006

[29] J. K. Riley, K. Takeda, S. Akira and R. D. Schreiber, "Interleukin-10 Receptor Signaling through the JAKSTAT Pathway. Requirement for Two Distinct ReceptorDerived Signals for Anti-Inflammatory Action,” The Journal of Biological Chemistry, Vol. 274, 1999, pp. 1651316521. http://dx.doi.org/10.1074/jbc.274.23.1651 